\title{
Production of Deuterated Cyanidin 3-O-Glucoside from Recombinant Escherichia coli
}

\author{
Mamta Gupta, ${ }^{\dagger, \ddagger}$ Jian Zha, ${ }^{\dagger}$ Xing Zhang, ${ }^{\dagger}$ Gyoo Yeol Jung, ${ }^{\S, \|_{\odot}}$ Robert J. Linhardt, ${ }^{\dagger}$ \\ and Mattheos A. G. Koffas* ${ }^{*}+\infty$
}

${ }^{\dagger}$ Department of Chemical and Biological Engineering, Center for Biotechnology and Interdisciplinary Studies, Rensselaer Polytechnic Institute, Troy, New York 12180, United States

${ }^{\ddagger}$ Department of Botany and Environment Studies, DAV University, Jalandhar 144 001, Punjab, India

${ }^{\S}$ Department of Chemical Engineering and "School of Interdisciplinary Bioscience and Bioengineering, Pohang University of Science and Technology, 77 Cheongam-ro, Nam-gu, Pohang, Gyeongbuk 37673, Korea

\section{Supporting Information}

ABSTRACT: Anthocyanins are plant secondary metabolites that, despite their chemical instability, have found many applications as natural food colorants. They are also known for their beneficial health effects because of their antioxidant and anticancer properties. More stable versions of these molecules, particularly at neutral $\mathrm{pH}$ conditions, are required to study the anthocyanin pharmacokinetic properties and obtain effective therapeutic results. In the present report, a cost-effective technique was developed to prepare the deuterated anthocyanin using recombinant Escherichia coli as a production host and deuterated glycerol and $\mathrm{D}_{2} \mathrm{O}$ in the culture media. This approach resulted in the formation of endogenous deuterated uridine 5'-diphosphateglucose that was further incorporated by the recombinant anthocyanin pathway, resulting in the formation of deuterated cyanidin 3-O-glucoside (C3G). The deuterium exchange of $\mathrm{O}-\mathrm{D}$ and $\mathrm{C}-\mathrm{D}$ were studied by liquid chromatography (LC)-mass spectrometry and NMR analysis. The labeled C3G, purified by high-performance LC showed a stable nature at $\mathrm{pH} 7.0$ as compared to nondeuterated C3G.

\section{INTRODUCTION}

Anthocyanins are a group of plant natural products and dietary polyphenols that are responsible, to a large extent, for the red color in fruits, vegetables, flowers, and red wine. ${ }^{1}$ Among these natural products, cyanidin 3-O-glucoside $(\mathrm{C} 3 \mathrm{G})$ is one of the most prevalent anthocyanins in nature. Because of the increasing preference for natural food colorants, anthocyanins in general and $\mathrm{C} 3 \mathrm{G}$ in particular have received considerable attention in recent years as candidates for replacing artificial food colorants and cosmetic additives. ${ }^{2-5}$ In addition, C3G has gained attention for its potential therapeutic role related to its neuroprotective effects, including protection against amyloid$\beta$-peptide-induced toxicity, ${ }^{6}$ antioxidant and cognitive promotion activity, ${ }^{7}$ anti-ischemic, anticancer, and anti-inflammatory properties. $^{8,9}$

Because of their promising therapeutic potential, various pharmacokinetic studies have focused on analyzing the signaling mechanisms of anthocyanins such as $\mathrm{C} 3 \mathrm{G}$ in vital organs. ${ }^{10-12}$ Unfortunately, the disappearance of C3G in the blood stream is very fast owing to its rapid biotransformation and its chemical instability at normal $\mathrm{pH}^{10}$ As a result, there is considerable interest in using more stable anthocyanins, such as deuterated anthocyanin derivatives. The biological effect of

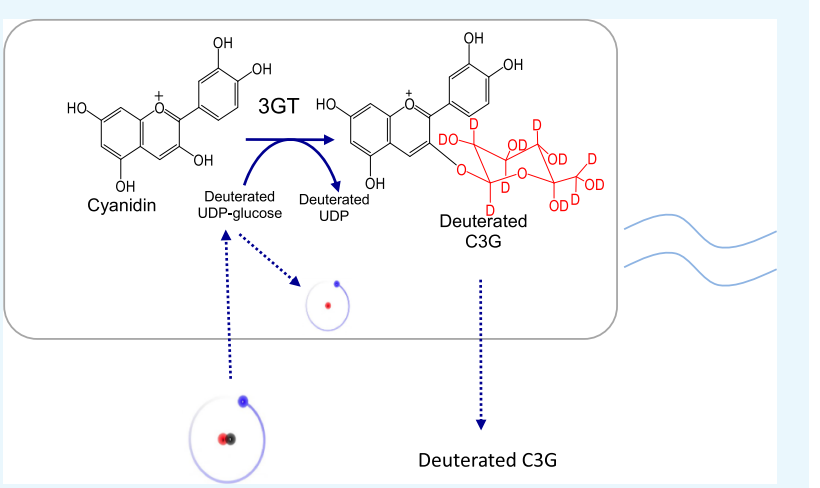

deuterium $\left({ }^{2} \mathrm{H}\right)$, with twice the mass of a hydrogen atom, has been extensively studied. Deuterium-carbon bonds are generally about six to 10 times more stable than the corresponding hydrogen-carbon bonds. As a result, they demonstrate slower rates of bond cleavage. The presence of deuterium also results in a kinetic isotope effect, ${ }^{13}$ which has been used to slow the metabolism of drugs when they reach vital organs and get metabolized through $\mathrm{C}-\mathrm{H}$ bond breaking. One or more well-placed deuterium can actually have a significant effect on how long a drug will circulate in the bloodstream, by slowing down its clearance. Deuterated compounds also can provide critical insights into the pharmacokinetic properties of molecules. ${ }^{14}$

As current methods of anthocyanin production rely on extraction from plant sources, they are not amenable to modifications that can allow the facile production of nonnatural anthocyanin derivatives, such as deuterated anthocyanins. However, in the recent past, microbial production of recombinant anthocyanins has emerged as an attractive

Received: May 25, 2018

Accepted: September 7, 2018

Published: September 24, 2018 


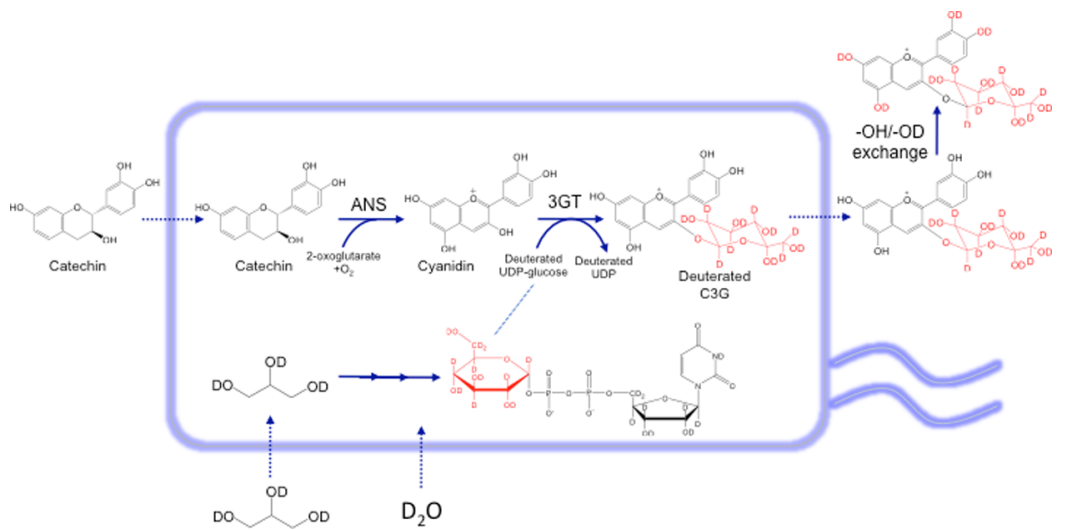

Figure 1. Schematic illustration of the biosynthesis of deuterated C3G by an engineered E. coli strain expressing ANS and flavonoid 3glucosyltransferase (3GT) in this study. Deuterated glycerol and $\mathrm{D}_{2} \mathrm{O}$ were used to support cell growth and to supply deuterated UDP-glucose, which provided the deuterated glucosyl group in the deuterated C3G. Regular catechin was employed as the substrate for the formation of the tricyclic structure in the deuterated $\mathrm{C} 3 \mathrm{G}$. The $-\mathrm{OH}$ group in the tricyclic structure can be exchanged with the deuterated hydroxyl $(-\mathrm{OD})$ of $\mathrm{D}_{2} \mathrm{O}$ in the medium.
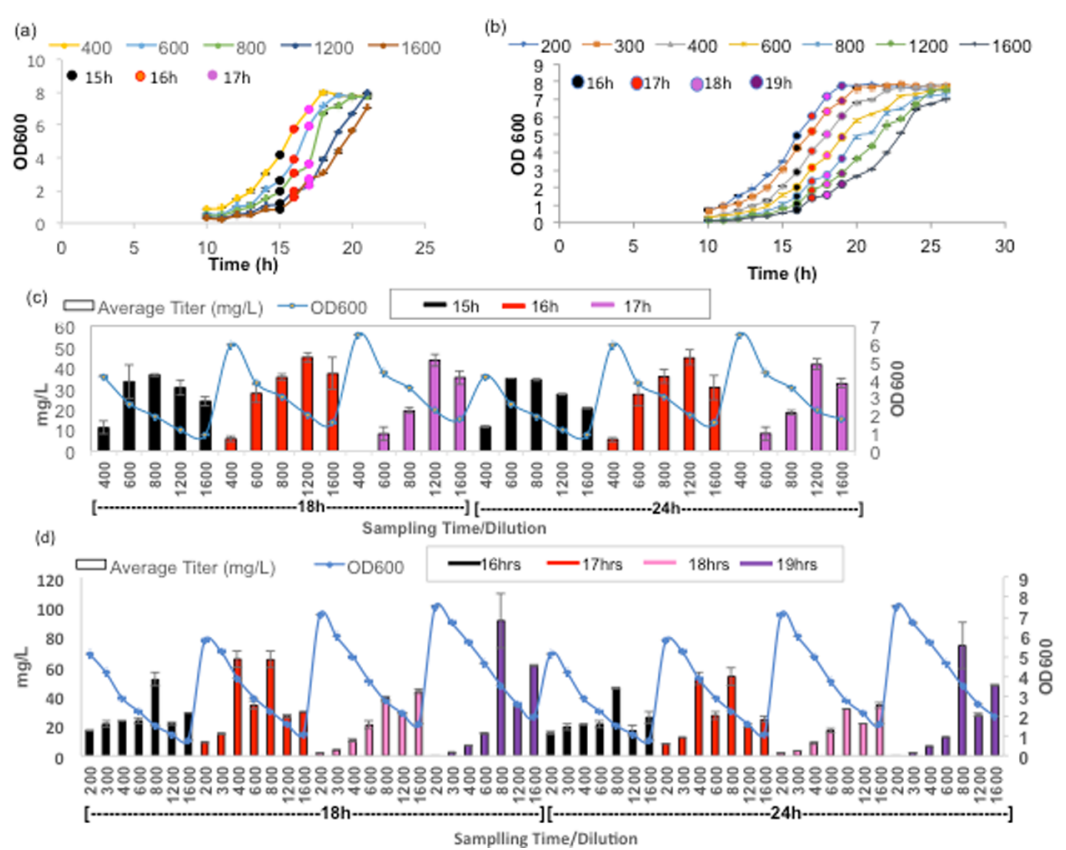

Figure 2. Growth curve of E. coli (At3GT + PhANS) at different dilutions in AMM supplemented with glucose (a) and glycerol (b) verifying different induction time points. Dynamics of production of C3G at different induction time points with sampling at 18 and $24 \mathrm{~h}$ in glucosesupplemented media (c) and glycerol-supplemented media (d). The values are average of three replicates. Error bars represent standard deviation.

alternative and more flexible production method, especially using well-characterized microbial hosts such as Escherichia coli. $^{15}$ In our laboratory, we have reconstituted the metabolic pathways leading to the biosynthesis of various anthocyanins, including $\mathrm{C} 3 \mathrm{G}$, from flavan-3-ol precursors as well as glucose. $^{16,17}$ We have shown that recombinant $E$. coli expressing anthocyanidin synthase (ANS) are able to convert the cheap and abundant precursor $(+)$-catechin into the unstable compound cyanidin, which can be significantly stabilized by glycosylation with uridine 5 -diphosphate (UDP)-glucose: 3-O-glycosyltransferase (3GT) to form C3G (Figure 1). In this work, we describe the biosynthesis of deuterated $\mathrm{C} 3 \mathrm{G}$ through recombinant E. coli as a production host by supplementing deuterated glycerol and deuterated water into the culture media (Figure 1). This is the first time the synthesis of a deuterated anthocyanin molecule has been reported, opening the possibility of synthesizing not only other deuterated anthocyanin molecules but also, more generally, deuterated polyphenols.

\section{RESULTS AND DISCUSSION}

Comparison of Glucose and Glycerol as Carbon Sources in C3G Fermentation. Glucose is the most widely utilized carbon source in industrial E. coli fermentations, ${ }^{18}$ but the excess glucose supplementation under aerobic conditions causes the formation of acidic byproducts by E. coli, the most common of which is acetate. ${ }^{19,20}$ Alternatively, glycerol can serve as an excellent carbon source despite the fact that it is a more energy-poor carbon source, and is a better source to produce regular and deuterated $\mathrm{C} 3 \mathrm{G}$ and has increased importance in industrial biotechnology. ${ }^{21,22}$ E. coli can utilize glucose and glycerol as carbon sources to produce destination 


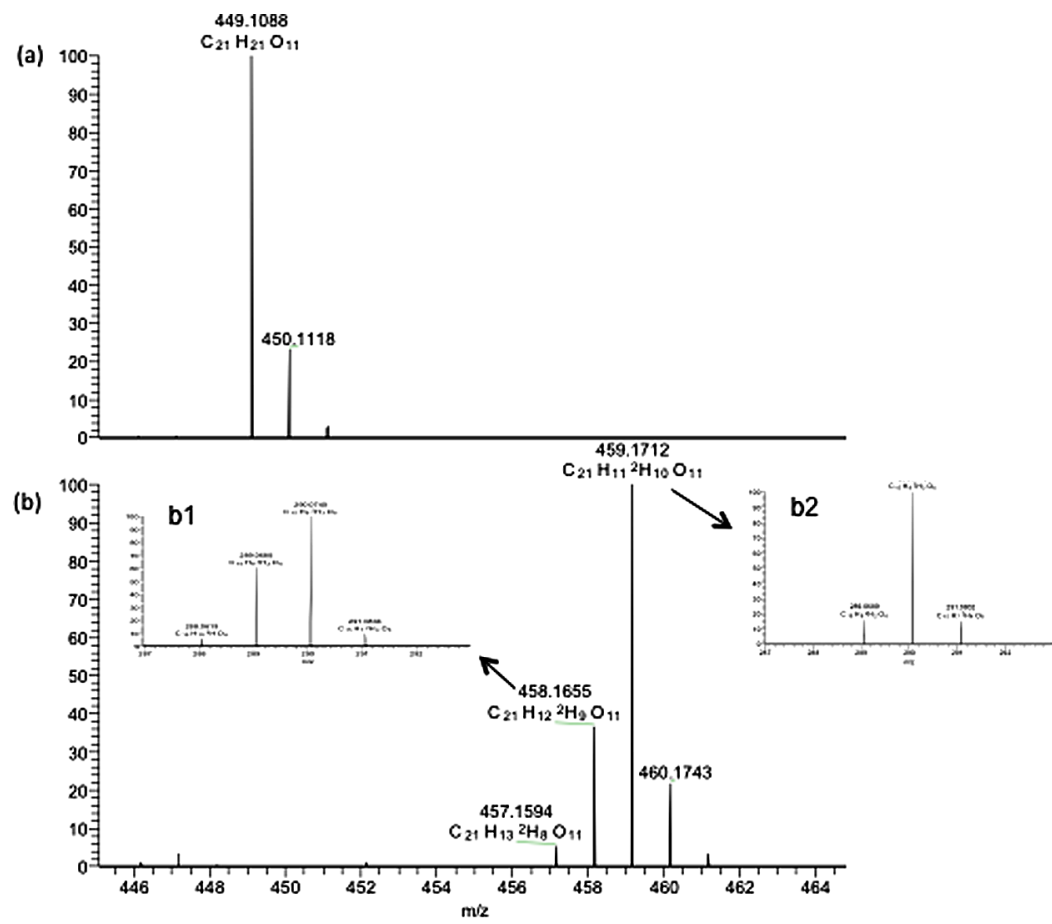

Figure 3. Fourier transform MS ESI positive-ion mode chromatogram of anthocyanidin glucoside; (a) nondeuterated sample; (b) deuterated sample; (b1,b2) MSMS ESI of the selected peak after losing glucoside residue shows different possibilities of deuteration in the aromatic ring.

compounds. Previously, our lab reported the production of flavan-3-ols using recombinant E. coli cocultures with different ratios of glycerol and glucose as a carbon source. ${ }^{23}$ In that work, increasing the glycerol percentage in the fermentation medium resulted in a shift in the production landscape toward higher titers appearing at later induction points. However, the effects of glycerol on $\mathrm{C} 3 \mathrm{G}$ production by E. coli have not been tested so far, to the best of our knowledge. Therefore, in the present study, to select a better carbon source for C3G production, we decided to compare the $\mathrm{C} 3 \mathrm{G}$ production by recombinant $E$. coli in the presence of both glucose and glycerol.

The growth of the C3G-producing strain was slower on glycerol-supplemented media than glucose (Figure S1). The recombinant strain reached the midlog phase $\left(\mathrm{OD}_{600} \approx 3.7\right)$ in $22 \mathrm{~h}$ after the start of the fermentation when grown in glycerolsupplemented media (Figure S1c), whereas it reached the same growth stage faster $\left(17 \mathrm{~h}, \mathrm{OD}_{600} \approx 3.5\right)$ in glucosesupplemented media (Figure S1b). This difference may be due to the incorporation of glycerol into the central metabolism as dihydroxyacetone phosphate that can participate in both gluconeogenic and glycolytic processes. ${ }^{24}$ Growth on glycerol resulted in a decrease of specific growth rate and low level of acetate production. ${ }^{25}$ The induction time points with isopropyl $\beta$-D-1-thiogalactopyranoside (IPTG) were also optimized for different cell dilutions separately in each glucose- and glycerolsupplemented Aspergillus minimal medium (AMM) $[3.5 \mathrm{~g} / \mathrm{L}$ $\mathrm{KH}_{2} \mathrm{PO}_{4}, 5.0 \mathrm{~g} / \mathrm{L} \mathrm{K} \mathrm{K}_{2} \mathrm{HPO}_{4}, 3.5 \mathrm{~g} / \mathrm{L}\left(\mathrm{NH}_{4}\right)_{2} \mathrm{HPO}_{4}, 100 \mathrm{~mL}$ 10× 3-(N-morpholino)propanesulfonic acid) (MOPS) mix (83.72 g/L MOPS, $7.17 \mathrm{~g} / \mathrm{L}$ tricine, $28 \mathrm{mg} / \mathrm{L} \mathrm{FeSO}_{4} \cdot 7 \mathrm{H}_{2} \mathrm{O}$, $29.2 \mathrm{~g} / \mathrm{L} \mathrm{NaCl}, 5.1 \mathrm{~g} / \mathrm{L} \mathrm{NH} \mathrm{NH}_{4}, 1.1 \mathrm{~g} / \mathrm{L} \mathrm{MgCl}_{2}, 0.48 \mathrm{~g} / \mathrm{L}$ $\mathrm{K}_{2} \mathrm{SO}_{4}$, and $0.2 \mathrm{~mL}$ micronutrient stock), $1 \mathrm{~mL} 1 \mathrm{M} \mathrm{MgSO}_{4}$, $0.1 \mathrm{~mL} 1 \mathrm{M} \mathrm{CaCl} 2$, and $1 \mathrm{~mL} 0.5 \mathrm{~g} / \mathrm{L}$ thiamine $\mathrm{HCl}$. Micronutrient stock contains $0.18 \mathrm{~g} / \mathrm{L}\left(\mathrm{NH}_{4}\right)_{6} \mathrm{Mo}_{7} \mathrm{O}_{24}, 1.24 \mathrm{~g} /$ $\mathrm{L}_{3} \mathrm{BO}_{3}, 0.12 \mathrm{~g} / \mathrm{L} \mathrm{CuSO}_{4}, 0.8 \mathrm{~g} / \mathrm{L} \mathrm{MnCl}_{2}$, and $0.14 \mathrm{~g} / \mathrm{L}$
$\mathrm{ZnSO}_{4}$, supplemented with $20 \%$ (w/v) glucose or glycerol with ampicillin] (Figure 2c,d). The objective of optimization was to achieve the maximum C3G titer by optimizing induction time points and sampling conditions. In glucose-supplemented media, the cells were induced at 15,16 , and $17 \mathrm{~h}$ after subculturing and sampling was done 18 and $24 \mathrm{~h}$ after induction. Induction at $16 \mathrm{~h}$ after subculturing resulted in maximum C3G production followed by 17 and $15 \mathrm{~h}$ time points. Overall, the cells with dilutions of 1200 and 1600 showed better results than lower dilutions. The highest titer achieved was $44.98 \mathrm{mg} / \mathrm{L}$, at $\mathrm{OD}_{600} \approx 2.8$ with induction at 16 $\mathrm{h}$ after inoculation, and sampled $24 \mathrm{~h}$ following induction (Figure 2c).

In glycerol-supplemented media, the cultures were induced at later induction time points, that is, 17,18 , and $19 \mathrm{~h}$ at different cell dilutions (Figure 2d). The induction at $19 \mathrm{~h}$ after subculturing showed enhanced production of C3G at 800 and 1600 dilutions. Sampling at $18 \mathrm{~h}$ after induction proved to be better than $24 \mathrm{~h}$ sampling. The highest titer of C3G was obtained with glycerol as the sole carbon source and was 90.95 $\mathrm{mg} / \mathrm{L}$ at $\mathrm{OD}_{600} \approx 3.7$ by inducing the recombinant $\mathrm{C} 3 \mathrm{G}$ pathway $19 \mathrm{~h}$ after inoculation, and sampled $18 \mathrm{~h}$ after induction (Figure 2d).

The better C3G production in low-cost glycerol-supplemented media than glucose-supplemented media allowed us to continue with the production of deuterated anthocyanins by using deuterated glycerol as the carbon source in the medium.

Deuterium-Labeled C3G Production. The deuteriumlabeled C3G production was carried out in AMM where normal water and normal glycerol were replaced by deuterated water $\left(\mathrm{D}_{2} \mathrm{O}\right)$ and deuterated glycerol- $d_{8}$. Liquid chromatography-mass spectrometry (LC-MS) analysis showed the different probabilities of deuterium incorporation to the $\mathrm{C} 3 \mathrm{G}$ compound in the culture supernatant (Figure $3 \mathrm{~b}$ ), whereas no deuteration was found in $\mathrm{C} 3 \mathrm{G}$ produced in normal medium 
(Figure 3a). The highest probability of deuterium incorporation to $\mathrm{C} 3 \mathrm{G}$ was of 10 atoms of deuterium (mass 459) followed by nine atoms of deuterium (mass 458) (Figure 3b). The MS/MS spectrum of the selected peak clearly confirmed most of the deuterium incorporation in the glucose residue of C3G, which is consistent with the preliminary design that deuterium in deuterated glycerol will be incorporated into UDP-glucose and delivered into the glucose residue in deuterated $\mathrm{C} 3 \mathrm{G}$, as regular catechin was used as the precursor for the formation of the tricyclic structure of C3G. Thus, the deuterated hydrogens in deuterated C3G are mainly located in the glycosyl group. The deuterium was also incorporated into the tricyclic ring, which was possibly ascribed to the deuterium-hydrogen exchange in the presence of $\mathrm{D}_{2} \mathrm{O}$.

For structural determination of deuterated C3G, NMR analysis was performed. Deuterated C3G was purified from the crude sample using high-performance LC (HPLC) (Figure S2) and subject to NMR analysis. Treating regular anthocyanins with $1 \% \mathrm{DCl}$ in $500 \mu \mathrm{L} \mathrm{MeOD}-d_{4}$ for $1 \mathrm{~h}$, the subsequent ${ }^{1} \mathrm{H}$ NMR spectrum was exactly matched with the commercial C3G anthocyanin standard (Figure S3). This control experiment demonstrated that hydrogen-deuterium exchange on the carbon atoms from glucose residue of anthocyanins did not occur under the fermentation conditions. Characterization of the purified deuterated anthocyanins and regular anthocyanins by NMR spectroscopy is presented in Figure 4. Peaks at 5.0-

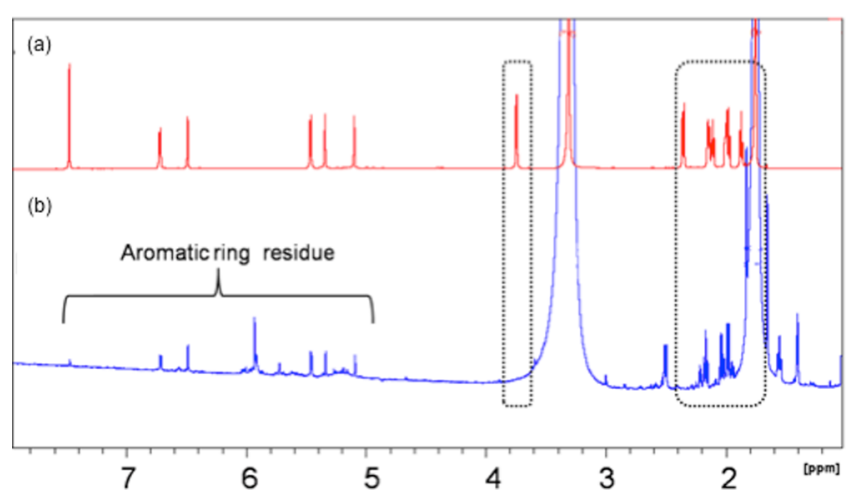

Figure 4. ${ }^{1} \mathrm{H}$ NMR comparison between commercial anthocyanins (a) and deuterated anthocyanins (b). Peaks at 5.0-7.5 ppm (denoted with a bracket) in both spectra correspond to aromatic protons on anthocyanins and are exactly matched. Peaks at 1.8-4.0 ppm (denoted in dashed boxes) in panel A correspond to protons from glucose residue but did not show up in panel B, demonstrating that deuterated exchange happened on glucose residue.

$7.5 \mathrm{ppm}$ (denoted with a bracket) in both spectra correspond to aromatic protons on anthocyanins and are exactly matched, indicating that no hydrogen-deuterium exchange occurred on the aromatic ring. Peaks at $1.8-4.0 \mathrm{ppm}$ (denoted in dashed boxes) in panel (a) correspond to protons of carbon atoms from glucose residue, such as peak at $3.8 \mathrm{ppm}$ that corresponds to glucose-anomeric hydrogen, but did not show up in panel (b), demonstrating that hydrogen-deuterium exchange happened on glucose residue. This technique is very promising to produce labeled anthocyanins having a much stronger $\mathrm{C}-\mathrm{D}$ bond, the content of which can be measured in the tissues during pharmacokinetic studies using heteronuclear NMR.

Stability Analysis of Deuterated C3G. At normal pH, anthocyanins are highly unstable molecules that are susceptible to degradation in response to different environmental factors such as oxygen, temperature, and light. ${ }^{26}$ Anthocyanins are more stable at acidic $\mathrm{pH}(1-3)$. In aqueous solutions at $\mathrm{pH}>$ 4 , anthocyanins adopt the form of carbinol and chalcones that undergo degradation easily and produce phenolic acids. ${ }^{27}$ The degradation of anthocyanins at normal $\mathrm{pH}$ is an important factor that can influence their therapeutic properties, given that the $\mathrm{pH}$ of the human blood is around 7.3 and at $7.3{ }^{10}$ More generally, it plays a critical role in determining the application of these molecules in the food industry as natural food colorants. Therefore, it was necessary to test the stability of the produced deuterated $\mathrm{C} 3 \mathrm{G}$ at normal $\mathrm{pH}$.

The $\mathrm{pH}$ stability test of deuterated and nondeuterated $\mathrm{C} 3 \mathrm{G}$ was performed at $\mathrm{pH} 7.0$ and showed a more stable nature for deuterated $\mathrm{C} 3 \mathrm{G}$ as compared to normal $\mathrm{C} 3 \mathrm{G}$ at room temperature (Figure 5). The sample analysis by HPLC at

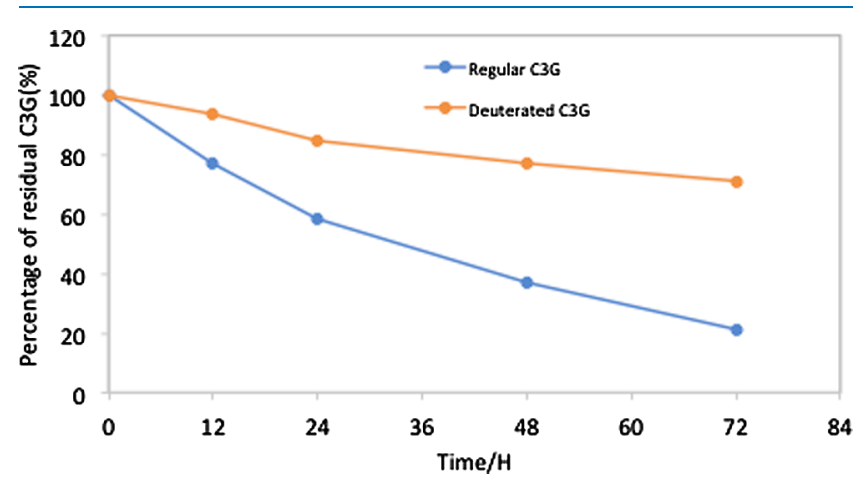

Figure 5. Stability comparison between regular C3G (blue line) and deuterated C3G (red line) at different intervals of time (from 0 to 72 h) at $\mathrm{pH} 7.4$, demonstrating early fall for regular $\mathrm{C} 3 \mathrm{G}$ as compared to deuterated $\mathrm{C} 3 \mathrm{G}$.

regular intervals showed more decrease in residual percentage of regular $\mathrm{C} 3 \mathrm{G}$ (from 100 at $0 \mathrm{~h}$ to $21.2 \%$ at $72 \mathrm{~h}$ ) as compared to deuterated C3G (from 100 at $0 \mathrm{~h}$ to $71 \%$ at 72 h).

The improved stability of deuterated C3G will result in longer residence times of this molecule in the human body, possibly resulting in increased absorption, distribution, metabolism, and excretion in clinical trials. We envision that deuterated $\mathrm{C} 3 \mathrm{G}$ can be used to better study the fate of anthocyanins in human bodies and to investigate their interaction with human cells.

\section{CONCLUSIONS}

In the present study, recombinant $E$. coli was used to convert deuterated glycerol and $\mathrm{D}_{2} \mathrm{O}$ to deuterated UDP-glucose that was incorporated into the deuterated $\mathrm{C} 3 \mathrm{G}$ by the last step in the biosynthetic pathway, catalyzed by the glycosyltransferase 3GT. The biosynthesis process presented in this work is benign and cost-effective. Therefore, recombinant E. coli strains can provide a very convenient and effective approach to synthesizing deuterated $\mathrm{C} 3 \mathrm{G}$, which only requires simple deuterated glycerol and $\mathrm{D}_{2} \mathrm{O}$ in the fermentation medium. Such deuterated anthocyanins can be used in the future in pharmacokinetic studies in animal models. Moreover, this technique can also be applied for the production of other deuterated anthocyanins using engineered E. coli incorporated with other anthocyanins producing genes. 


\section{MATERIALS AND METHODS}

Strains and Chemicals. E. coli strains and plasmids used in this study were constructed previously in our lab, ${ }^{16}$ and are available through Addgene (plasmids \#86901-86917). The culture media and other chemicals, viz., LC-MS grade methanol, water, formic acid, MeOD- $d_{4}$ (99.9\%), deuterated glycerol- $d_{8}(99 \%)$, and deuterium chloride (99 atom \% D), were purchased from Sigma-Aldrich.

Bacterial Culture Conditions and Growth Curve. All anthocyanin production experiments were performed using a recombinant E. coli BL21 Star (DE3) carrying plasmid pETM6-At3GT-m-PhANS where a two-step anthocyanin pathway has been introduced in monocistronic configuration. ${ }^{16}$ The working culture was maintained on Luria-Bertani agar plates supplemented with $80 \mu \mathrm{g} / \mathrm{mL}$ ampicillin. All growth as well as production experiments were performed in $1 \mathrm{~mL}$ AMM as described previously in polypropylene deep 48-well plates (5 $\mathrm{mL}, \mathrm{VWR}$ ) covered with breathable rayon film (VWR). Casamino acids were excluded as they are a potential carbon source. ${ }^{17,28}$ For carrying out experiments in deuterated media, buffer salts and mineral salts were pre-equilibrated with $99.9 \%$ $\mathrm{D}_{2} \mathrm{O}$ and then flash-evaporated/lyophilized to dryness to remove exchangeable hydrogen atoms. The exchanged salts were then dissolved in $99.9 \% \mathrm{D}_{2} \mathrm{O}$ and filter-sterilized. Deuterated glycerol- $d_{8}(20 \mathrm{~g} / \mathrm{L})$ was used as a carbon source.

For obtaining a bacterial growth curve, glycerol stocks of recombinant BL21 Star (DE3) strains carrying anthocyanin pathway plasmids were grown on solid media and after overnight growth, a single colony was inoculated into a single well of a polypropylene deep 48-well plate containing $1 \mathrm{~mL}$ of liquid media. The plate was then incubated at $37{ }^{\circ} \mathrm{C}$ with shaking at $225 \mathrm{rpm}$. Optical cell density was measured on a BioTek Synergy 4 Microplate Reader.

Optimization of Induction Time Points. For induction time point optimization in glucose-supplemented media after $17 \mathrm{~h}$ of overnight growth, 400-, 600-, 800-, 1200-, and 1600fold dilutions were made, whereas for glycerol-supplemented media after 22 h of overnight growth, 200-, 300-, 400-, 600-, $800-, 1200-$, and 1600-fold dilutions were made. All cultures were incubated at $225 \mathrm{rpm}$ and $30{ }^{\circ} \mathrm{C}$. Substrate $(1 \mathrm{~g} / \mathrm{L}$ (+)-catechin final concentration) stock dissolved in dimethylformamide/ethanol $(8: 2, \mathrm{v} / \mathrm{v})$ and inducer ( $1 \mathrm{mM}$ IPTG final concentration) was added sequentially by multichannel pipette at different time points after subculturing. These time points were $15,16,17 \mathrm{~h}$ for glucose (Figure $2 \mathrm{a}$ ) and 16, 17, 18, and $19 \mathrm{~h}$ for glycerol-supplemented media (Figure 2b). Cultures were sampled for anthocyanin quantification at 18 and $24 \mathrm{~h}$ postinduction. All production experiments were performed in biological triplicate, and reported production values represent mean and standard error of the mean as quantified by HPLC.

HPLC Analysis and Purification. The $200 \mu \mathrm{L}$ of cell culture was extracted by addition of an equal volume of acidified methanol $(1 \% \mathrm{HCl}, \mathrm{v} / \mathrm{v})$, followed by brief vortexing and centrifugation at $21000 \mathrm{~g}$ for $10 \mathrm{~min}$. C3G stock solutions of known concentrations were prepared by dissolving analytical standards (purchased from Extrasynthese) in dimethyl sulfoxide and were stored at $-20{ }^{\circ} \mathrm{C}$. Standard curves for quantifying the anthocyanidin glucoside production were prepared by dilution of $\mathrm{C} 3 \mathrm{G}$ into methanol at appropriate concentrations. The samples or standards $(10 \mu \mathrm{L})$ were injected for analysis on an Agilent 200 series HPLC equipped with a ZORBAX SB-C18 StableBond analytical column (150 $\mathrm{mm} \times 4.6 \mathrm{~mm}, 5 \mu \mathrm{m})$ and a diode array detector. Acetonitrile (solvent A) and water (solvent B), both containing $0.1 \%$ formic acid, were used as mobile phases. A flow rate of $1 \mathrm{~mL} /$ min was used with the following gradients: $10-40 \%$ A (0-10 $\mathrm{min})$ and $10-40 \% \mathrm{~A}(10-15 \mathrm{~min})$. C3G was quantified at a retention time of $4.8 \mathrm{~min}$ against the standard curves by peak area integration at $520 \mathrm{~nm}$.

Anthocyanins were purified from substrate catechin as well as other components of media using HPLC with $60 \mu \mathrm{L}$ injection volume. The separation was conducted using mobile phases $\mathrm{A}$ and $\mathrm{B}$ as defined above, at a flow rate of $1 \mathrm{~mL} / \mathrm{min}$ with the following gradients: $1.0-15 \%$ A (0-45 $\mathrm{min}), 15-$ $60 \%$ A (45-50 min), 60-1.0\% (50-52 $\mathrm{min}$ ), and 1\% A (52$55 \mathrm{~min})$.

LC-MS Analysis. LC analysis was performed by injecting 5 $\mu \mathrm{L}$ of the sample to the Agilent 1200 HPLC system with column Agilent ZORBAX Eclipse XDB-C18 $4.6 \times 150 \mathrm{~mm} 5$ $\mu \mathrm{m}$. Water and acetonitrile containing $0.2 \%$ formic acid were used as solvents $\mathrm{A}$ and $\mathrm{B}$, respectively, at a flow rate of $250 \mu \mathrm{L} /$ min, using the following gradients: $5-10 \% \mathrm{~B}(0-1 \mathrm{~min}), 10-$ $30 \%$ B (1-10 min), $30-90 \%$ B (10-30 min), $90-5 \%$ B (34.9-35 $\mathrm{min})$, and 5\% B (35-40 min). MS analysis was done using LTQ Orbitrap XL from Agilent Technologies (Santa Clara, CA, USA), electrospray ionization (ESI), positive-ion mode, resolution 30 000, and mass accuracy 3 ppm.

NMR Analysis. All samples were dissolved in $0.5 \mathrm{~mL}$ of MeOD- $d_{4}$ (contains $1 \% \mathrm{DCl}$ ). ${ }^{1} \mathrm{H}$ spectra were performed at $298 \mathrm{~K}$ on a Bruker $800 \mathrm{MHz}$ instrument recorded for 32 scans with TopSpin 2.1.6 software.

Stability Test of Deuterated Anthocyanins. The purified deuterated $\mathrm{C} 3 \mathrm{G}$ or regular C3G (Alkemist Labs, USA) was dissolved in phosphate buffered saline ( $\mathrm{pH} 7.4$ ) with an initial concentration of $\sim 20 \mathrm{mg} / \mathrm{L}$ and was left at room temperature in the dark for $72 \mathrm{~h}$. Samples were taken periodically and the residual C3G was measured by HPLC.

\section{ASSOCIATED CONTENT}

\section{Supporting Information}

The Supporting Information is available free of charge on the ACS Publications website at DOI: 10.1021/acsomega.8b01134.

Structure of anthocyanidin glucosides (PDF)

\section{AUTHOR INFORMATION}

\section{Corresponding Author}

*E-mail: koffam@rpi.edu.

ORCID $\odot$

Gyoo Yeol Jung: 0000-0002-9742-3207

Robert J. Linhardt: 0000-0003-2219-5833

Mattheos A. G. Koffas: 0000-0002-1405-0565

Notes

The authors declare no competing financial interest.

\section{ACKNOWLEDGMENTS}

The authors are grateful to the SERB Indo-US Science and Technology Forum for their financial support. This research is also supported by the NSF CBET award number 1604547 and by the Global Research Laboratory Program (NRF 2016K1A1A2912829) through the National Research Foundation of Korea (NRF). 


\section{REFERENCES}

(1) Wu, X.; Beecher, G. R.; Holden, J. M.; Haytowitz, D. B.; Gebhardt, S. E.; Prior, R. L. Concentrations of Anthocyanins in Common Foods in the United States and Estimation of Normal Consumption. J. Agric. Food Chem. 2006, 54, 4069-4075.

(2) Tanaka, Y.; Ohmiya, A. Seeing Is Believing: Engineering Anthocyanin and Carotenoid Biosynthetic Pathways. Curr. Opin. Biotechnol. 2008, 19, 190-197.

(3) Ananga, A.; Georgiev, V.; Ochieng, J.; Phills, B.; Tsolov, V. Production of Anthocyanins in Grape Cell Cultures: A Potential Source of Raw Material for Pharmaceutical, Food, and Cosmetic Industries. In The Mediterranean Genetic Code-Grapevine and Olive; Poljuha, D., Sladonja, B., Eds.; InTech, 2013; pp 247-287.

(4) Aberoumand, A. A review article on edible pigments properties and sources as natural biocolorants in foodstuff and food industry. World J. Dairy Food Sci. 2011, 6, 71-78.

(5) Bhan, N.; Xu, P.; Koffas, M. A. G. Pathway and Protein Engineering Approaches to Produce Novel and Commodity Small Molecules. Curr. Opin. Biotechnol. 2013, 24, 1137-1143.

(6) Tarozzi, A.; Morroni, F.; Merlicco, A.; Bolondi, C.; Teti, G.; Falconi, M.; Cantelli-Forti, G.; Hrelia, P. Neuroprotective Effects of Cyanidin 3-O-Glucopyranoside on Amyloid Beta (25-35) OligomerInduced Toxicity. Neurosci. Lett. 2010, 473, 72-76.

(7) Shih, P.-H.; Chan, Y.-C.; Liao, J.-W.; Wang, M.-F.; Yen, G.-C. Antioxidant and cognitive promotion effects of anthocyanin-rich mulberry (Morus atropurpurea L.) on senescence-accelerated mice and prevention of Alzheimer's disease. J. Nutr. Biochem. 2010, 21, $598-605$.

(8) Kang, T. H.; Hur, J. Y.; Kim, H. B.; Ryu, J. H.; Kim, S. Y. Neuroprotective effects of the cyanidin-3-O- $\beta$-d-glucopyranoside isolated from mulberry fruit against cerebral ischemia. Neurosci. Lett. 2006, 391, 122-126.

(9) Di Giacomo, C.; Acquaviva, R.; Santangelo, R.; Sorrenti, V.; Vanella, L.; Li Volti, G.; D’Orazio, N.; Vanella, A.; Galvano, F. Effect of Treatment with Cyanidin-3-O- $\beta$-D-Glucoside on Rat Ischemic/ Reperfusion Brain Damage. Evidence-Based Complementary Altern. Med. 2012, 2012, 1-10.

(10) Fornasaro, S.; Ziberna, L.; Gasperotti, M.; Tramer, F.; Vrhovšek, U.; Mattivi, F.; Passamonti, S. Determination of Cyanidin 3-Glucoside in Rat Brain, Liver and Kidneys by UPLC/MS-MS and Its Application to a Short-Term Pharmacokinetic Study. Sci. Rep. 2016, 6, 22815.

(11) Frank, T.; Sonntag, S.; Strass, G.; Bitsch, I.; Bitsch, R.; Netzel, M. Urinary Pharmacokinetics of Cyanidin Glycosides in Healthy Young Men Following Consumption of Elderberry Juice. Int. J. Clin. Pharmacol. Res. 2005, 25, 47-56.

(12) Jeon, S.; Han, S.; Lee, J.; Hong, T.; Yim, D.-S. The Safety and Pharmacokinetics of Cyanidin-3-Glucoside after 2-Week Administration of Black Bean Seed Coat Extract in Healthy Subjects. Korean J. Physiol. Pharmacol. 2012, 16, 249-253.

(13) Guengerich, F. P. Kinetic Deuterium Isotope Effects in Cytochrome P450 Reactions. Methods Enzymol. 2017, 596, 217-238.

(14) Kushner, D. J.; Baker, A.; Dunstall, T. G. Pharmacological Uses and Perspectives of Heavy Water and Deuterated Compounds. Can. J. Physiol. Pharmacol. 1999, 77, 79-88.

(15) Pandey, R. P.; Parajuli, P.; Koffas, M. A. G.; Sohng, J. K. Microbial Production of Natural and Non-Natural Flavonoids: Pathway Engineering, Directed Evolution and Systems/Synthetic Biology. Biotechnol. Adv. 2016, 34, 634-662.

(16) Cress, B. F.; Leitz, Q. D.; Kim, D. C.; Amore, T. D.; Suzuki, J. Y.; Linhardt, R. J.; Koffas, M. A. G. CRISPRi-Mediated Metabolic Engineering of E. Coli for O-Methylated Anthocyanin Production. Microb. Cell Factories 2017, 16, 10.

(17) Zhao, S.; Jones, J. A.; Lachance, D. M.; Bhan, N.; Khalidi, O.; Venkataraman, S.; Wang, Z.; Koffas, M. A. G. Improvement of Catechin Production in Escherichia Coli through Combinatorial Metabolic Engineering. Metab. Eng. 2015, 28, 43-53.
(18) Gosset, G. Improvement of Escherichia Coli Production Strains by Modification of the Phosphoenolpyruvate:Sugar Phosphotransferase System. Microb. Cell Factories 2005, 4, 14.

(19) Luli, G. W.; Strohl, W. R. Comparison of Growth, Acetate Production, and Acetate Inhibition of Escherichia Coli Strains in Batch and Fed-Batch Fermentations. Appl. Environ. Microbiol. 1990, 56, 1004-1011.

(20) Wang, J.; Zhu, J.; Bennett, G. N.; San, K.-Y. Succinate Production from Different Carbon Sources under Anaerobic Conditions by Metabolic Engineered Escherichia Coli Strains. Metab. Eng. 2011, 13, 328-335.

(21) Bisen, P. S.; Sanodiya, B. S.; Thakur, G. S.; Baghel, R. K.; Prasad, G. B. K. S. Biodiesel Production with Special Emphasis on Lipase-Catalyzed Transesterification. Biotechnol. Lett. 2010, 32, 1019-1030.

(22) Yazdani, S. S.; Gonzalez, R. Anaerobic Fermentation of Glycerol: A Path to Economic Viability for the Biofuels Industry. Curr. Opin. Biotechnol. 2007, 18, 213-219.

(23) Jones, J. A.; Vernacchio, V. R.; Sinkoe, A. L.; Collins, S. M.; Ibrahim, M. H. A.; Lachance, D. M.; Hahn, J.; Koffas, M. A. G. Experimental and Computational Optimization of an Escherichia Coli Co-Culture for the Efficient Production of Flavonoids. Metab. Eng. 2016, 35, 55-63.

(24) Martínez-Gómez, K.; Flores, N.; Castañeda, H. M.; MartínezBatallar, G.; Hernández-Chávez, G.; Ramírez, O. T.; Gosset, G.; Encarnación, S.; Bolivar, F. New Insights into Escherichia Coli Metabolism: Carbon Scavenging, Acetate Metabolism and Carbon Recycling Responses during Growth on Glycerol. Microb. Cell Factories 2012, 11, 46.

(25) Peng, L.; Shimizu, K. Global Metabolic Regulation Analysis for Escherichia Coli K12 Based on Protein Expression by 2-Dimensional Electrophoresis and Enzyme Activity Measurement. Appl. Microbiol. Biotechnol. 2003, 61, 163-178.

(26) Fernandes, I.; Faria, A.; Calhau, C.; de Freitas, V.; Mateus, N. Bioavailability of Anthocyanins and Derivatives. J. Funct. Foods 2014, 7, 54-66.

(27) Fang, J. Bioavailability of Anthocyanins. Drug Metab. Rev. 2014, $46,508-520$.

(28) Jones, J. A.; Vernacchio, V. R.; Collins, S. M.; Shirke, A. N.; Xiu, Y.; Englaender, J. A.; Cress, B. F.; McCutcheon, C. C.; Linhardt, R. J.; Gross, R. A.; Koffas, M. A. G. Complete Biosynthesis of Anthocyanins Using E. coli Polycultures. mBio 2017, 8, e00621. 